\title{
Unsupervised free-breathing 3D imaging of morphology, function and flow in congenital heart disease
}

\author{
Rajesh Krishnamurthy ${ }^{1,2^{*}}$, Ramkumar Krishnamurthy ${ }^{1}$, LaDonna Malone ${ }^{1}$, Elijah Bolin², Amol Pednekar ${ }^{3}$ \\ From 17th Annual SCMR Scientific Sessions \\ New Orleans, LA, USA. 16-19 January 2014
}

\section{Background}

To evaluate technical feasibility, image quality and quantitative integrity of a free-breathing protocol following administration of blood pool contrast agent, utilizing 3-dimensional (3D) imaging of morphology, function, and flow without physician supervision in a cohort of patients with CHD.

\section{Methods}

Five patients with CHD were included in this pilot study. The MR studies were performed on a Philips Acheiva $1.5 \mathrm{~T}$ magnet using a multielement phased array coil with the following sequences in this order: 1 . Free-breathing, respiratory synchronized [1], time-resolved MRA following injection of $0.03 \mathrm{mmol} / \mathrm{kg}$ of Gadofosveset, and injection rate $2-4 \mathrm{cc} /$ second using a power injector. Duration: $<1$ minute 2 . Free breathing equilibrium phase MRA, acquired voxel size $0.8 \times 0.8 \times 1.6 \mathrm{~mm}, 2 \mathrm{NEX}$. Duration: 2.5-4 minutes 3. Free breathing 3D cine SSFP with respiratory triggering (TR/TE/flip angle: 3/1.5/60; acquired voxel size: 1.5-1.9 $\times 1.5-2.1 \times 7-8 \mathrm{~mm} 3$; SENSE acceleration factor: $1.5-2 \times 1.5-2$; temporal resolution: 30-45 ms). Duration: 4.5-7 minutes 4 . Free breathing sagittal 4D phase contrast (PC) imaging with respiratory navigator (18-26 phases/cardiac cycle, Venc $150 \mathrm{~cm} / \mathrm{sec}$, spatial resolution 1.6-2.8 mm3.) Duration: 6-12 minutes 5. Free breathing 3D SSFP with respiratory navigator. (acquired voxel size $1 \times 1 \times 2 \mathrm{~mm} 3$ ) Duration: 5-7 minutes Comparative data was obtained using conventional 2D cine respiratory triggered SSFP sequences (2) in the VLA, 4 chamber and short axis planes, and 2D PC imaging. Data Analysis: Image quality assessment and quantitative volumetric and flow analysis was performed by a single blinded user. MRA images were graded using a semi-quantitative scale from 1-5 for relevant imaging targets in CHD [1], with 1: excellent, no limitations, and 5: non-diagnostic. The clinical scoring system for $2 \mathrm{D}$ and $3 \mathrm{D}$ cine SSFP was based on blood-myocardial contrast, endocardial edge definition and inter-slice alignment[2]. Paired t-test analysis was performed on LV and RV volumes.

\section{Results}

All free-breathing 3D sequences were technically feasible in all 5 patients. Average time for performance of 5 free breathing 3D sequences was 29 minutes. Average score for first-pass MRA was 1.9/5. Average score for equilibrium MRA was 1.3/5. Clinical scores for 2D SSFP were consistently better than 3D-SSFP, but 3D SSFP images were adequate for recognition of pathology in all cases. Average percentage difference between 2D and 3D cine SSFP volumetric data were as follows: LVEDV (5.5\%), LVSV (11.2\%), LVEF (6\%), RVEDV (-3.9\%), RVSV (8\%) and RVEF (9.1\%). Comparative flow analysis between 2D PC and 4D PC data is pending.

\section{Conclusions}

It is feasible to perform an observer independent comprehensive CMR in CHD utilizing free-breathing 3D acquisitions for morphology, function and flow within 30 minutes.

\footnotetext{
Authors' details

${ }^{1}$ Radiology, TexasChildren's Hospital, Houston, Texas, USA. ${ }^{2}$ Pediatrics, Baylor College of Medicine, Houston, Texas, USA. ${ }^{3}$ Research Scientist, Philips Medical Systems, Houston, Texas, USA.
} 
Published: 16 January 2014

\section{References}

1. JCMR 2010, 12(Suppl 1):031.

2. JCMR 2013, 15(Suppl 1):098.

doi:10.1186/1532-429X-16-S1-P134

Cite this article as: Krishnamurthy et al:: Unsupervised free-breathing 3D imaging of morphology, function and flow in congenital heart disease. Journal of Cardiovascular Magnetic Resonance 2014 16(Suppl 1):P134.

Submit your next manuscript to BioMed Central and take full advantage of:

- Convenient online submission

- Thorough peer review

- No space constraints or color figure charges

- Immediate publication on acceptance

- Inclusion in PubMed, CAS, Scopus and Google Scholar

- Research which is freely available for redistribution

Submit your manuscript at www.biomedcentral.com/submit
C Biomed Central 